\title{
Diabetes and Stress: A Review
}

\author{
Analava Mitra \\ School of Medical Science and Technology, Indian Institute of Technology, Kharagpur 721 30, \\ West Bengal, India \\ Telephone: 91-322-282220/282657(R), Fax: 91-322-282221 \\ E-mail: amitra@adm.iitkgp.ernet.in
}

KEYWORDS Stress; diabetes; fight or flight response; fast-paced society

ABSTRACT Stress is a part and parcel of modern day life. Stressors may be internal and external. Our body responds to stress either as fight or flight response and repeated stress may lead to failing rheostat phenomenon of hypothalamus leading to less efficient hormonal control through feed-backs. This leads to various changes in body functioning at various levels like cellular, organic or systemic and finally leads to various diseases. Diabetes may be an outcome of stress and further sets in a vicious cycle of stress-diabetes relationship. Stress coping mechanisms are many and it depends on resources available with a wide range of personal variations.

\section{INTRODUCTION}

\section{Definition of Stress and Stressors}

It's hard to dispute that most of us live life at breakneck speed. It's the nature of a fast-paced society, where numerous family, social, and work obligations can easily overpower precious time and resources. But for people with diabetes both physical and emotional stress can take a greater toll on health. Stress is a feeling that's created when one reacts to particular events. It's the body's way of rising to a challenge and preparing to meet a tough situation with focus, strength, stamina, and heightened alertness. Stress results when something causes the body to behave as if it were under attack. The events that provoke stress are called stressors, and they cover a whole range of situations like physical, injury or illness. Or they can be mental, like problems in marriage, job, health, or finances (Meadows-Oliver et al., 2007).

\section{Definition of Diabetes}

The oxford dictionary defines diabetes as an increase in urination and presently two types of diabetes are known-diabetes mellitus, that is, diabetes due to metabolic causes and diabetes insipidus, that is, diabetes due to pituitary dysfunctions. Diabetes mellitus being more common and literally means diabetes until classified otherwise. Of the various types of diabetes two types are more common- Type 1 and Type 2 . Type 2 diabetes or commonly known as maturity onset diabetes accounts for $80 \%$ of diabetic populations in most countries. Hence diabetes when unclassified means Type 2 diabetes.

\section{U.S. Public Health Survey Estimate of Stress}

A U.S. Public Health Survey estimated that 70 percent to 80 percent of Americans experience at least "some stress" every two weeks and visit a physician each year for a stress-related disorder. Job-related stress costs U.S. businesses $\$ 60$ million annually (DiClemente, 2006).

\section{Fight-or-Flight Response}

The body gears up to take action in response to stress. This preparation is called the fight-orflight response (ADA, 2007). Repeated stress may lead to failing rheostat phenomenon of hypothalamus leading to less efficient hormonal control through feed-back mechanisms (Dilman, 1986).

\section{Endocrine Changes}

In the fight-or-flight response, levels of many hormones shoot up. The hypothalamus signals the adrenal glands to produce more of the hormones catecholamines and cortisol and also stimulate pituitary gland to produce more growth hormone and release them into the bloodstream. These hormones speed up heart rate, breathing rate, blood pressure, and metabolism including coping of growth in case of injury related damage. 
Their net effect is to make a lot of stored energy, glucose and fat available to cells. This provides fuel for what has been traditionally referred to as the 'flight or fight' response. Insulin is not always able to let the extra energy into the cells, so glucose piles up in the blood. Further gonodal hormones are suppressed with a view that reproduction should not occur in a living under stress (Dilman, 1989).

\section{Physiological Changes}

Blood vessels open wider to let more blood flow to large muscle groups, putting our muscles on alert. Pupils dilate to improve vision. The liver releases some of its stored glucose to increase the body's energy. Sweat is produced to cool the body (Kahn and Weir, 1996). All of these physical changes prepare a person to react quickly and effectively to handle the pressure of the moment. Blood clotting mechanisms are more efficient with a tilt towards pro-coagulation and tissue reactions are towards damage recovery. The stress response can cause problems when it overreacts or fails to turn off and reset itself properly. People who are experiencing stress overload may notice some of the following signs (Wyngaarden et al., 1988):

- Anxiety or panic attacks

- A feeling of being constantly pressured, hassled, and hurried

- Irritability and moodiness

- Sadness or depression

- Problems in sleeping

- Drinking too much, smoking, overeating, or taking drugs

- Physical symptoms, such as stomach problems, headaches, or even chest pain

- Allergic reactions, such as eczema or asthma Such constant stress can also add to a variety of other health concerns from ulcers to high blood pressure

- It can also result in changes to emotions and to the ways in which one behave that might not be good for his diabetes

- Integrated electromyographic activity of the temporalis muscle, but not the masseter muscle, showed an increase that coincided with the increase in sympathetic nervous activity (Hidaka et al., 2004)

\section{Stress Related Disorders}

Stress can lead to disorders including (Sudhalter, 2007):

\begin{abstract}
Headaches
- Viral infections

- Heart disease, high cholesterol and arterial disorders

- Digestive disorders

- Asthma and allergies

- Cancer

- Adult-onset diabetes

- Memory impairment

According to the Occupational Stress Research Institute, stress costs U.S. industry some $\$ 150$ billion a year.

The immediate effects of stress are usually temporary and vary in severity from person to person. But over time, they can damage heart, arteries, brain, kidneys and eyes. Stress can suppress immune system, making one more likely to "catch" cold or contract more serious diseases.
\end{abstract}

\section{Metabolic Strategy of Stressed Cell}

Dynamic genetic and biochemical changes make up a cell's response to a shortage of Coenzyme A (CoA), a key player in metabolism. Coupled with genetic studies of the cell, metabolomics is giving scientists a more detailed picture of how the body maintains its health in both normal environments and during times of stress, such as starvation or disease. The response observed to decreased $\mathrm{CoA}$ in a mouse model by blocking CoA production with hopantenate (HoPan) was studied. HoPan is a chemical that interferes with pantothenate kinase (PanK), the enzyme that triggers the first step of $\mathrm{CoA}$ production. Following the shutdown of $\mathrm{CoA}$ production, the cells quickly recycled CoA from other sources, extracting life-supporting energy from nutrients in the mitochondria. Low levels of CoA trigger the activation of genes that block other biochemical pathways that ordinarily use this molecule. Instead, the cell shifts most of the available CoA activity to producing glucose from the liver. Other organs then break down glucose into .pyruvate inside mitochondria. In the mitochondria, CoA molecules feed pyruvate into a complex series of chemical reactions that produces molecules of ATP. Results identify the re-arrangements that the cell's metabolism undergoes in order to ensure that the liver keeps CoA levels high enough to produce glucose and the cells of the body maintain enough free $\mathrm{CoA}$ for the mitochondria to keep producing ATP (St. Jude Children's Research Hospital, Memphis: Tennessee, 2007). 


\section{STRESS AND DIABETES}

There is no evidence that stress causes diabetes. However, stress may sometimes unmask diabetes, by causing blood glucose levels to rise (Kahn and Weir, 1996). This is often seen after a heart attack or stroke, where raised blood sugar levels may be encountered for the first time. In people who have diabetes, the fight-or-flight response does not work well. Insulin is not always able to let the extra energy into the cells, so glucose piles up in the blood (ADA, 2007). Making things worse, many sources of stress are not short-term threats. For example, it can take many months to recover from surgery. Stress hormones that are designed to deal with short-term danger stay turned on for a long time. As a result, long-term stress can cause long-term high blood sugar levels. Many long-term sources of stress are mental. Like physical stress, mental stress can be short term from taking a test to getting stuck in a traffic jam. It can also be long term, from working for a demanding boss to take care of an aging parent. In mental stress, the body pumps out hormones to no avail. Physical stress, such as illness or injury, causes higher blood glucose levels in people with either Type of diabetes. Stress blocks the body from releasing insulin in people with Type 2 diabetes. The diagnosis of diabetes usually comes as a shock and is certainly a stressful time (Wijenaike, 2002; ADA, 2007). Changes in lifestyle including stoppage of smocking, diet and learning to manage injections may all contribute in addition to the worry regarding chronic illness (Davis et al., 1999).

In people who have diabetes, stress can alter blood sugar levels. It does this in two ways. First, people under stress may not take good care of themselves. People who are anxious are under pressures and may lose appetite and skimp on eating, or reach for not-so healthy quick fixes like candy or chips and sometimes seek refuge in food and drink. This can take the form of chocolates, sweets and crisps, often in between meals. The intake of alcohol may be increased. Many people who are under stress turn to food as a source of 'comfort'. This pattern of 'comfort eating' can often play havoc with blood sugar level. Further anxiety leads to less exercise. The results can be disastrous for people with diabetes. They may forget, or not have time, to check their sugar levels or plan good meals. Second, stress hormones may also alter blood sugar levels directly as it antagonizes the action of insulin. While in most people glucose levels go up with mental stress, while in others can go down. Diabetic mice under physical or mental stress have elevated glucose levels. The effects in people with Type 1 diabetes are more mixed. People with Type 1 diabetes may develop elevated blood glucose levels and ketoacidosis. Those with Type 2 diabetes usually gain weight and develop obesity and often blood sugar levels are raised (ADA, 2007). Inflammatory signaling pathways can also become activated by metabolic stresses originating from inside the cell as well as by extra-cellular signaling molecules. It has been demonstrated that obesity overloads the functional capacity of the endoplasmic reticulum and that this endoplasmic reticulum stress leads to the activation of inflammatory signaling pathways and thus contributes to insulin resistance. Additionally, increased glucose metabolism can lead to a rise in mitochondrial production of reactive oxygen species. Reactive oxygen species production is elevated in obesity, which causes enhanced activation of inflammatory pathways (Wellen and Hotamisligil, 2005).

Physical stress, such as illness or injury, causes higher blood sugar levels in people with either type of diabetes. For some people with diabetes, controlling stress with relaxation therapy seems to help. It is more likely to help people with Type 2 diabetes than people with Type 1 diabetes. Stress blocks the body from releasing insulin in people with Type 2 diabetes, so cutting stress may be more helpful for these people. People with Type 1 diabetes do not make insulin, so stress reduction does not have this effect. Reducing stress can help people with Type 1 diabetes take better care of them. Some people with Type 2 diabetes may also be more sensitive to some of the stress hormones. Relaxing can help by blunting this sensitivity. In people with Type 2 diabetes, mental stress often raises blood glucose levels. It is easy to find out whether mental stress affects glucose control. Many glucose meters have the capability to enter personal notes and data when one perform checks, or jot it down in a stress journal (Wijenaike, 2002). Once one begins recording stress levels, most people with diabetes figure out pretty quickly what makes his blood sugar to go up. People with diabetes should stay conscious of eating well and exercising regularly. It's a good idea to check blood glucose levels more frequently 
when ill or under stress and to drink plenty of fluids as so as not to get dehydrated. Something else that affects peoples responses to stress coping style. Coping style is how a person deals with stress. People who use them tend to have less blood sugar elevation in response to mental stress (ADA, 2007).

Hyperglycemia induces the overproduction of oxygen free radicals and consequently increases the protein oxidation and lipid oxidation. A significance difference in the mean plasma concentration of total antioxidant status was observed in diabetic patients. A statistically significant higher values of protein carbonyl groups and MDA as lipid peroxides were observed in diabetic patients with slight reduction in the synthesis of nitric oxide. It is interesting to note that there was a decrease in the antioxidant levels with corresponding increased protein and lipid oxidation. Decreased levels of proteins albumin, transferrin, ceruloplasmin and heptoglobulins and variable GC globulin fractions in diabetes were found compared to normal healthy controls (Vadde and Jailkhani, 2007).

\section{DEALING WITH SUCCESS}

The most helpful method of dealing with stress is learning how to manage the stress that comes along with any new challenge, good or bad. Stress-management skills work best when they're used regularly, not just when the pressure's on. Knowing how to "de-stress" and doing it when things are relatively calm can help one get through challenging circumstances that may arise. Here are some things that can help keep one to keep stress under control (Strickland et al., 2007; Wijenaike 2002; Heiden et al., 2007; Meadows-Oliver et al., 2007)

- Regular courses of yoga, meditation or deep breathing

- Progressive relaxation therapy in which one practices tensing and relaxing major muscle groups in sequence

- Cognitive behaviour therapy by helping one to change his behaviour and teaching to view the life through more appropriately colored glasses-to take a stand against over-scheduling, be realistic, solving the little problems, to watch what one is thinking

- Talking to a therapist

- Step back from the situation

- To keep up healthy eating and exercise routine
To get a good night's sleep

To eliminate Caffeine

Anti-anxiety medication

To take up a relaxing hobby

Replace bad thoughts with good ones

Biofeedback training

Massage

- Other ways to reduce mental stress- outlook, attitude, and thoughts influence the way to see things. A healthy dose of optimism can help one to make the best of stressful circumstances.

- Building Resilience

- Thinking of change as a challenging and normal part of life

- To see setbacks and problems as temporary and solvable

- To believe that one will succeed if he keeps working toward his goals

- To take action to solve problems that crop up

- To build strong relationships and keep commitments to family and friends

- To have a support system and ask for help

- To participate regularly in activities for relaxation and fun

- Tai Chi- Tai Chi chuan (Tai Chi) is an ancient, widely practiced Chinese martial arts form. Like yoga, it is designed to enhance both physical and emotional well-being (Sudhalter, 2006).

- A sniff of lavender- Aromatherapy, which relies on the use of essential volatile oils of flowers and fruits to cause biochemical effects, has been proven to have positive effects on the mood. When it comes to stress, lavender is supposed to be particularly uplifting (Sudhalter, 2006).

\section{CONCLUSION}

It's hard to dispute that most of us live life at breakneck speed. Stress is a feeling that's created when one reacts to particular events. It's the body's way of rising to a challenge and preparing to meet a tough situation with focus, strength, stamina, and heightened alertness. The events that provoke stress are called stressors, and they cover a whole range of situations- physical, like injury or illness. Or they can be mental, like problems in marriage, job, health, or finances. The body gears up to take action in response to stress. This preparation is called the fight or flight response. In the fight or flight response, levels of many hormones like catecholamines, cortisol and growth hormone shoot up. Their net effect is to 
make a lot of stored energy, glucose and fat available to cells. Insulin is not always able to let the extra energy into the cells, so glucose piles up in the blood. These results in increase propensity of various diseases and diabetes may be an outcome of stress, which further sets in a vicious cycle of stress-diabetes relationship. The most helpful method of dealing with stress is learning how to manage the stress that comes along with any new challenge, good or bad. Stress-management skills work best when they're used regularly, not just when the pressure's on. Knowing how to "de-stress" and doing it when things are relatively calm can help one get through challenging circumstances that may arise.

\section{REFERENCES}

American Diabetes Association. Official Home Page of American Diabetes Association. 2007. How Stress Affects Diabetes. Retrieved on 14. 2. 2007 from http://www.diabetes.org/type-1-diabetes/stress.jsp.

Davis, R. M., E. H. Wagner and T. Groves. 1999. "Management of Chronic Diseases." British Medical Journal, 318: 1090-1.

Dilman, V. M. 1989. The Grand Biological Clock. Moscow: Mir Publishers.

Heiden, M., E. Lyskov, M. Nakata, K. Sahlin, T. Sahlin and M. Barnekow-Bergkvist, M. May 2007.

"Evaluation of cognitive behavioural training and physical activity for patients with stress-related illnesses: a randomized controlled study." Journal of Rehabilitation Medicine, 39(5): 366-73.

Hidaka, O., M. Yanagi and K. Takada. 2004. "Mental Stress-induced Physiological Changes in the Human Masseter Muscle." Journal of Dental Research, 83(3): 227-231.
Huang, C. Y., V. D. Sousa, H. F. Chen, S. Y. Tu, C. J. Chang and I. J. Pan. 2007. "Stressors, depressive symptoms, and learned resourcefulness among Taiwanese adults with diabetes mellitus." Research and Theory for Nursing Practice, 21(2): 83-97.

Kahn, C. R. and G. C. Weir. (Eds.). 1996. Joslincs Diabetes Mellitus. New Delhi: B.I. Waverly Pvt. Ltd.

Meadows-Oliver, M., L. S. Sadler, M. K. Swartz and P. Ryan-Krause. 2007. "Sources of stress and support and maternal resources of homeless teenage mothers." Journal of Child and Adolescent Psychiatric Nursing, 20(2): 116-25.

St. Jude Children's Research Hospital, Memphis: Tennessee. Official Home Page of St. Jude Children's Research Hospital. March 23, 2007. "Study shows metabolic strategy of stressed cell." Retrieved on 26. 6. 2007 from http://www.stjude.org.

Strickland, O. L., J. N. Giger, M. A. Nelson and C. M. Davis. 2007. "The relationships among stress, coping, social support, and weight class in premenopausal African American women at risk for coronary heart disease." Journal of Cardiovascular Nursing, 22(4): 272-8.

Sudhalter, Vivian. Official Home Page of health a to $\mathrm{z}$. com. 2007. "Relaxing Ways to Ease Stress." Retrieved on 14. 6. 2007 from https://www.healthatoz.com

Vadde, Ramakrishna and Rama Jailkhani. 2007. "Evaluation of oxidative stress in Insulin Dependent Diabetes Mellitus (IDDM) patients." Diagnostic Pathology, doi:10.1186/1746-1596-2-22.

Wellen, E. Kathryn and S. Gökhan.Hotamisligil. 2005. "Inflammation, stress, and diabetes." The Journal of Clinical Investigation, 115:1111-1119.

Wijenaike, Nishan. Official Home Page of Diabete Suffolk. com. 23 ${ }^{\text {rd }}$ November 2002. "Diabetes and Stress". Retrieved on 22. 3. 2007 from http://www. diabetesuffolk.com.

Wyngaarden, J. B., L. H. Smith and J. C. Bennett. (Eds.). 1988. Cecil Textbook of Medicine. London W. B. Saunders Company. 